\title{
Prevalencia de maloclusiones en escolares de 6 y 12 años del cantón Biblián. Ecuador
}

\section{Prevalence of malocclusions in school children aged 6 and 12 from the Biblián canton. Ecuador}

\author{
Ana María Ormaza-Zamora ${ }^{1 *}$, José Fernando Tintín-Rea $^{1}$, Mariela delCarmen Ramírez-Velásquez ${ }^{1}$ \\ ${ }^{1}$ Universidad Católica de Cuenca \\ *amormazaz40@est.ucacue.edu.ec
}

DOI: https://doi.org/10.26871/killkana_salud.v4i1.583

\begin{abstract}
Resumen
Objetivo: Determinar la prevalencia de maloclusiones en escolares de 6 y 12 años de las escuelas Héroes de Verdeloma y Daniel Muñoz del Cantón Biblián durante el período 2018. Materiales y método: se realizó una investigación observacional de tipo transversal y descriptiva, aplicada a una muestra de 281 escolares de 6 y 12 años de edad, conformada por 165 varones y 116 mujeres, los cuales fueron evaluados clínicamente según la clasificación de Angle, determinando su relación molar. Resultados: Los resultados evidencian una prevalencia de maloclusión de 49,8\%, mientras que el $50,2 \%$ presentó normoclusión o relación molar clase I bilateral. Según la edad, en los escolares de 6 años se determinó una prevalencia de $20,7 \%$ de maloclusiónes, mientras que en los de 12 años un 29,1\%, viéndose mayormente afectados; con una significancia estadística entre edad y maloclusiónes $(\mathrm{p}=0,005)$. Según el sexo, los escolares de sexo masculino evidenciaron un $28,9 \%$ de maloclusiones y los escolares del sexo femenino $21,2 \%$ de afectación; de acuerdo a su procedencia, los escolares de zonas rural evidenciaron mayor prevalencia de maloclusiones que los de procedencia urbana. En cuanto a la clase molar, la relación molar clase I fue la más prevalente, seguida de la relación molar clase III. Conclusiones: El estudio determinó que la mitad de la población estudiada presentó cierto grado de maloclusión, siendo la edad de 12 años, el sexo masculino y los escolares de procedencia rural los más afectados. La clase molar más prevalente fue la clase I seguida de la clase III.
\end{abstract}

Palabras clave: prevalencia, maloclusión, molar, escolares.

\begin{abstract}
Objective: To determine the malocclusion prevalence in 6 and 12-year-old schoolchildren from Héroes de Verdeloma and Daniel Muñoz Schools in Biblián, during the 2018 period. Materials and methods: An observational, cross-sectional and descriptive study was carried out and applied to a sample of 281 schoolchildren aged 6 and 12 years. One hundred and sixty five(165) participants were male, and 116 were female. They were clinically evaluated by using Angle's classification to determine their molar relation. Results: The results evidenced a malocclusion prevalence of 49,8\% whereas 50,2\% presented normocclusion, or bilateral Angle's Class I molar relation. Concerning age, $20.7 \%$ prevalence of malocclusion was shown in 6-year-old schoolchildren, against 29,1\% in 12 year-olds. The last ones were the most affected, with a statistical significance between age and malocclusions $(p=0,005)$. As regards sex, males presented $28.9 \%$ of malocclusion while females showed 21,2\% prevalence. According to their origin, schoolchildren from rural areas had higher malocclusions prevalence than those from the urban areas. As for the molar class, the molar class I ratio was the most prevalent, followed by molar class III. Conclusions: The study revealed that half of the studied population had some level of malocclusion, 12-year-olds, males, and rural schoolchildren were the most affected. The most prevalent was Angle class I, followed by Angle class III.
\end{abstract}

Keywords: prevalence, malocclusion, molar, schoolchildren.

\section{Introducción}

Las malolusiones son estimadas dentro de los problemas dentales, de aparición muy frecuente por la Organización Mundial de la salud (OMS) ${ }^{1}$

La salud bucodental es definida por la OMS como la ausencia de dolor orofacial, cáncer bucal, infecciones, enfermedades periodontales, caries, pérdida de dientes y otras enfermedades y trastornos que restringen en la persona afectada su capacidad de morder, masticar, sonreír y hablar, repercutiendo en su bienestar psicosocial. ${ }^{1}$ Las maloclusiones son alteraciones que pueden ocurrir durante el crecimiento y desarrollo del niño pudiendo afectar la for- 
ma de los arcos dentarios, además de las distintas funciones ejecutadas por el sistema estomatognático afectando la salud bucodental y la salud general de las personas.

Estas alteraciones se relacionan con problemas estéticos faciales que pueden ocasionar un impacto psicológico en los individuos sobre todo en adolescentes, ${ }^{2}$ además de problemas de disfunciones de la articulación temporomandibular $^{3}$ que pudieran llegar a limitar las funciones normales de masticación y fonación, etc. Pueden ocasionar además problemas posturales 4

Existen distintos criterios para evaluar la relación oclusal como la propuesta por Solow ${ }^{5}$ mas sin embargo la clasificación de Edwar Angle a inicios del siglo XX es referencia para diagnosticar las maloclusiones de origen dentario, basada en la relación de las cúspides de los primeros molares permanentes superiores e inferiores, esta clasifica las maloclusiones en clase I, II y III.

La prevalencia de maloclusiones se estima de un $65 \%$ hasta un $89 \%$ a nivel mundial; en Latinoamérica se han realizado diversos estudios resultando una prevalencia que supera el $80 \%,{ }^{67}$ En Ecuador un estudio ${ }^{8}$ realizado en la provincia de Azuay reportó una prevalencia de $85 \%$ de maloclusiones. Así mismo una investigación ${ }^{9}$ reportada en escolares de 12 años determinó una prevalencia de maloclusiónes tanto en el sexo femenino y masculino de 95,7\%. Astudillo ${ }^{10}$ por su parte reportó en Cuenca evidencia de una significativa prevalencia de maloclusiones en los escolares de 12 años de la parroquia Bellavista, siendo más frecuentes las maloclusiones en mujeres que en varones. Es de resaltar que no se evidencia resultados al respecto en la Provincia de Cañar; y debido a la importancia del tema por las consecuencias que pudiera traer en el sistema estomatognático y la salud general de los individuos son necesarias investigaciones que proporcionen un diagnostico al respecto; por lo cual este estudio tiene como objetivo determinar la prevalencia de maloclusiones en escolares de 6 y 12 años de las escuelas Héroes de Verdeloma y Daniel Muñoz del Cantón Biblián en la provincia del Cañar Ecuador.

\section{Materiales y Métodos}

\subsection{Tipo de investigación}

Se realizó un estudio observacional, transversal y descriptivo,

\subsection{Población y muestra}

La población de estudio estuvo conformada por 360 escolares de 6 y 12 años de edad matriculados en las escuelas Daniel Muñoz y Héroes de Verdeloma del cantón Biblián, durante el periodo 2018. La muestra final estuvo conformada por 281 escolares de ambos sexos que cumplieron con los siguientes criterios de inclusión: escolares de 6 y 12 años cumplidos al momento de la recolección de datos, con dentición mixta o permanente y con los cuatro primeros molares permanentes en boca. Se excluyeron a aquellos escolares que hayan recibido tratamiento ortodóntico previo, o sean portadores de aparatología ortodóntica u ortopédica. Inicialmente se solicitó el permiso de las diferentes instituciones educativas de la parroquia mediante la autorización emitida por el distrito zonal de educación Todos los padres y/o representantes de los niños que participaron en el estudio firmaron además un informe de consentimiento, luego de explicarles el propósito del estudio

\subsection{Determinación de la relación de oclusión}

El procedimiento se inició realizando el examen clínico visual con el escolar sentado frente al operador, bajo luz natural y con la ayuda de un espejo bucal $\mathrm{N}^{\circ} 5$, cuidando todos los aspectos relacionados a la asepsia y la antisepsia. Se determinó el tipo de maloclusión de acuerdo a la clasificación de Angle, ${ }^{11}$ identificando relaciones molares clase I, clase II, clase II división I y II, así como relaciones molares clase III con variaciones uni o bilaterales; para ello se le colocó al escolar un abrebocas, solicitandole que ocluyera como normalmente lo hace en máxima intercuspidación, se registraron todos los datos obtenidos de la exploración en el instrumento diseñado para tal fin, que constaba de 2 partes, la primera para datos generales del escolar como edad, sexo, institución a la que pertenecen y su procedencia; y la segunda parte registraba la relación molar según Angle.

\subsection{Análisis estadístico de los resultados}

Los datos obtenidos se analizaron en el programa SPSS versión 23, elaborando previamente una base de datos con el concerniente diccionario; para el estudio estadístico univariado, se obtuvieron tablas de frecuencias absolutas y relativas, y el análisis bivariado se efectuó a través la prueba de Chi cuadrado. El estudio contó con un nivel de confianza de $95 \%$ y un $\mathrm{p}<0.05$.

\section{Resultados}

Un total de 281 escolares de 6 y 12 años de edad fueron incluidos en el estudio, 80 escolares del sexo masculino de 6 años y 85 de 12 años representando el 28,5\% y 30,2\% respectivamente de la muestra total. El sexo femenino estuvo representado por 59 escolares de 6 años $(21 \%)$ y 57 de 12 años $(20,3 \%)$. En total la muestra estuvo representada por $58.7 \%$ escolares del sexo masculino y $41,3 \%$ escolares del sexo femenino.

En cuanto a la prevalencia de las maloclusiones se determinó que el $49,8 \%$ de escolares del sexo femenino y masculino de 6 y 12 años presentaron maloclusiones. (Tabla 1) 
Tabla 1. Prevalencia de maloclusiones en escolares de 6 y 12 años de edad de las escuelas Héroes de Verdeloma y Daniel Muñoz del cantón Biblián en el período 2018

\begin{tabular}{|l|c|c|}
\hline & $\mathrm{N}$ & $\%$ \\
\hline Clase I bilateral (normoclusión) & 141 & 50,2 \\
\hline Maloclusión & 140 & 49,8 \\
\hline Total & 281 & 100 \\
\hline
\end{tabular}

N: Frecuencia absoluta. \%: Frecuencia relativa.

Se determinaron además características específicas de la oclusión de los escolares de 6 y 12 años evaluados, donde el $29.1 \%$ de los escolares de 12 años presentaron maloclusiones clase II y III bilateral, y/o clase II div I y II y/o sus respectivas subdivisiones. Observandose además que los escolares de 6 años de edad presentaron en un 20,7\% maloclusiones varias, con sus características específicas descritas en la Tabla 2. Existe relación estadísticamente significativa entre edad y maloclusiones $(\mathrm{p}=0,005)$

Tabla 2. Maloclusiones según la relación molar de Angle en escolares de 6 y 12 años de edad de las escuelas Héroes de Verdeloma y Daniel Muñoz del cantón Biblián

\begin{tabular}{|c|c|c|c|c|c|c|c|}
\hline \multirow{3}{*}{ Clase de Angle } & \multicolumn{6}{|c|}{ Edad } & \multirow{3}{*}{$\mathrm{p}^{*}$} \\
\hline & \multicolumn{2}{|c|}{6 años } & \multicolumn{2}{|c|}{12 años } & \multicolumn{2}{|c|}{ total } & \\
\hline & $\mathrm{n}$ & $\%$ & $\mathrm{n}$ & $\%$ & $\mathrm{~N}$ & $\%$ & \\
\hline Clase I bilateral & 81 & 28,8 & 60 & 21,4 & 141 & 50,2 & 0,005 \\
\hline Clase II bilateral & 6 & 2,1 & 9 & 3,2 & 15 & 5,3 & \\
\hline Clase III bilateral & 44 & 15,7 & 46 & 16,4 & 90 & 32,0 & \\
\hline Clase II div. 1 & 1 & 0,4 & 10 & 3,6 & 11 & 4,0 & \\
\hline Clase II div. 2 & 7 & 2,5 & 6 & 2,1 & 13 & 4,6 & \\
\hline Clase I der-clase III izq. & 0 & 0,0 & 4 & 1,4 & 4 & 1,4 & \\
\hline Clase II der.-clase I izq. & 0 & 0,0 & 4 & 1,4 & 4 & 1,4 & \\
\hline Clase II der.-clase III izq. & 0 & 0,0 & 2 & 0,7 & 2 & 0,7 & \\
\hline Clase III der.-clase I izq. & 0 & 0,0 & 1 & 0,4 & 1 & 0,4 & \\
\hline Total & 139 & 49,5 & 142 & 50,5 & 281 & 100,0 & \\
\hline
\end{tabular}

N: Frecuencia absoluta. \%: Frecuencia relativa. p: Significancia estadística. *: Prueba de Chi-cuadrado

En cuanto a la prevalencia de maloclusiones distribuidas según sexo se observa que 28,9 de los escolares del sexo masculino presentaron maloclusiones mientras que solo el
$21,2 \%$ de escolares del sexo femenino estuvieron afectadas, como se puede observar en la Tabla 3

Tabla 3. Prevalencia de maloclusiónes en escolares de 6 y 12 años según sexo

\begin{tabular}{|c|c|c|c|c|c|c|c|}
\hline \multirow{3}{*}{ Clase de Angle } & \multicolumn{6}{|c|}{ Edad } & \multirow{3}{*}{$\mathrm{p}^{*}$} \\
\hline & \multicolumn{2}{|c|}{ Masculino } & \multicolumn{2}{|c|}{ Femenino } & \multicolumn{2}{|c|}{ total } & \\
\hline & $\mathrm{n}$ & $\%$ & $\mathrm{n}$ & $\%$ & $\mathrm{~N}$ & $\%$ & \\
\hline Clase I bilateral & 84 & 29,9 & 57 & 20,3 & 141 & 50,2 & 0,846 \\
\hline Clase II bilateral & 9 & 3,3 & 6 & 2,1 & 15 & 5,4 & \\
\hline Clase III bilateral & 51 & 18,1 & 39 & 13,9 & 90 & 32,0 & \\
\hline Clase II div. 1 & 6 & 2,1 & 5 & 1,8 & 11 & 3,9 & \\
\hline Clase II div. 2 & 6 & 2,1 & 7 & 2,5 & 13 & 4,6 & \\
\hline Clase I der.-clase III izq. & 3 & 1,1 & 1 & 0,4 & 4 & 1,4 & \\
\hline Clase II der-clase I izq. & 3 & 1,1 & 1 & 0,4 & 4 & 1,4 & \\
\hline Clase II der.-clase III izq. & 2 & 0,7 & 0 & 0,0 & 2 & 0,7 & \\
\hline Clase III der.-clase I izq. & 1 & 0,4 & 0 & 0,0 & 1 & 0,4 & \\
\hline Total & 165 & 58,8 & 116 & 41,2 & 281 & 100,0 & \\
\hline
\end{tabular}

N: Frecuencia absoluta. \%: Frecuencia relativa. p: Significancia estadística. *: Prueba de Chi-cuadrado

Se determinó también la prevalencia de maloclusiones según la procedencia de los escolares, bien de zonas rurales o urbanas, identificando a los escolares de las zonas urbanas con menor prevalencia de maloclusiones en un $19,9 \%$ mientras los escolares que procedían de zonas rurales, evidenciaron una prevalencia de un $30 \%$, como se observa en la Tabla 4 
Tabla 4. Prevalencia de maloclusión en escolares de 6 y 12 años según su lugar de procedencia

\begin{tabular}{|c|c|c|c|c|c|c|c|}
\hline \multirow{3}{*}{ Clase de Angle } & \multicolumn{6}{|c|}{ Edad } & \multirow{3}{*}{$\mathrm{p}^{*}$} \\
\hline & \multicolumn{2}{|c|}{ Urbana } & \multicolumn{2}{|c|}{ Rural } & \multicolumn{2}{|c|}{ total } & \\
\hline & $\mathrm{n}$ & $\%$ & $\mathrm{n}$ & $\%$ & $\mathrm{~N}$ & $\%$ & \\
\hline Clase I bilateral & 58 & 20,6 & 83 & 29,5 & 141 & 50,2 & 0,506 \\
\hline Clase II bilateral & 8 & 2,8 & 7 & 2,5 & 15 & 5,3 & \\
\hline Clase III bilateral & 38 & 13,5 & 52 & 18,5 & 90 & 32,0 & \\
\hline Clase II div. 1 & 3 & 1,1 & 8 & 2,9 & 11 & 4,0 & \\
\hline Clase I div. 2 & 6 & 2,1 & 7 & 2,5 & 13 & 4,6 & \\
\hline Clase I der--clase III izq. & 1 & 0,4 & 3 & 1,1 & 4 & 1,4 & \\
\hline Clase II der.-clase I izq. & 0 & 0,0 & 4 & 1,4 & 4 & 1,4 & \\
\hline Clase II der.-clase III izq. & 0 & 0,0 & 2 & 0,7 & 2 & 0,7 & \\
\hline Clase III der.-clase I izq. & 0 & 0,0 & 1 & 0,4 & 1 & 0,4 & \\
\hline Total & 114 & 40,5 & 167 & 59,5 & 281 & 100,0 & \\
\hline
\end{tabular}

N: Frecuencia absoluta. \%: Frecuencia relativa. p: Significancia estadística. *: Prueba de Chi-cuadrado

\section{Discusión}

Los resultados de este estudio, realizado en las escuelas Daniel Muñoz y Héroes de Verdeloma del cantón Biblián de la provincia del Cañar de Ecuador durante el período 2018, en 281 escolares de 6 y 12 años de ambos sexos y de procedencia tanto urbana como rural, permitieron determinar la prevalencia de maloclusión lo cual es considerada como un problema de salud pública. ${ }^{12}$

Cabe señalar, que la clasificación de Angle, es un método que permite un diagnóstico de maloclusiones a través de un análisis de relación molar en el plano anteroposterior, pero no identifica una maloclusión verdadera. Para ello se debe tomar en cuenta parámetros como las llaves de la oclusión dadas por Lawrence Andrews, relaciones verticales y transversales, discrepancias en línea media, etc. Sin embargo, los resultados obtenidos en esta investigación inicial proporcionan la prevalencia de las maloclusiones en la población estudiada permitiendo un estudio de diagnóstico base, para posteriormente determinar la severidad y necesidad de tratamiento de las mismas, en futuras investigaciones.

La prevalencia de maloclusiones en esta investigación fue de un $49,8 \%$ menor que la relación molar bilateral calificada como normoclusion que fue de $50,2 \%$, estos resultados se contraponen a los encontrados por Pincheira ${ }^{13}$ de la alta prevalencia de maloclusiones en escolares de 6 y 12 años en las localidades de Choshuenco y Neltume. Además, cabe acotar que los resultados de otros estudios realizados en la provincia de Azuay evidencian mayor prevalencia de maloclusiones en escolares de 12 años ${ }^{8910}$

Los resultados de este estudio determinaron una mayor prevalencia de maloclusiónes en los escolares de 12 años del $29,2 \%$, concordando con el estudio realizado por Pincheira $^{13}$ y cols., con una muestra de 91 escolares, en los cuales el $91.3 \%$ de los escolares de 12 años presentaron maloclusión.

En cuanto al sexo, los resultados de esta investigación demuestran mayor prevalencia de maloclusiones en el sexo masculino de un 28,9\% lo cual concuerda con los de Ramírez, ${ }^{14}$ quien reporta que en el $61 \%$ de los casos fue el sexo masculino quien resultó con mayor alteración en la fisiología oclusal. A diferencia de las evidencias obtenidas por Talley ${ }^{12}$ y cols.

De acuerdo a la relación molar según Angle la de mayor prevalencia según lo reportado en la investigación realizada por Medina ${ }^{15}$ estuvo representada por la clase I molar, seguida de la clase II, y clase III, similares resultados reporta Ruiz $^{16}$ y Talley. ${ }^{12} \mathrm{~A}$ diferencia de los resultados de este estudio los cuales determinaron una prevalencia mayor de relación molar clase I seguida de la relación molar clase III y siendo la de menor prevalencia la relación molar clase II, lo cual sirve de base para investigaciones futuras en virtud de conocer las causas de la alta prevalencia de maloclusiones clase III ya que el crecimiento y desarrollo del complejo cráneo facial está bajo control mutifactorial. Sin embargo, las influencias genéticas tienden a jugar siempre un papel dominante. ${ }^{17}$

\section{Fuente de Financiamiento}

Este estudio es autofinanciado.

\section{Conflicto de Intereses}

No existen conflictos personales, profesionales, financieroso de otro tipo.

\section{Consentimiento Informado}

Los autores cuentan con el consentimiento informado de los pacientes para la investigación, la publicación del caso y sus imágenes.

\section{Referencias Bibliográficas}

1. Organización Panamericana de la Salud. Perfiles básicos de salud de países en las Américas. Área Andina e Istmo Centroamericano. Organización Panamericana de la Salud. 2012; Volumen de los Países.

2. Calzada-Bandomo A, Mora-Pérez C, Calzada-Bandomo A, Álvarez-Mora I, Pérez-García R, Rodríguez-López J. Necesidad de tratamiento ortodóncico en escolares. Aplicación del índice de prioridad de tratamiento de Shaw. Revista Medisur. 2014;12(4):622-624. 
3. Manfredini D, Stellini E, Marchese-Ragona R, GuardaNardini L. Are occlusal features associated with different temporomandibular disorder diagnoses in bruxers? Cranio. 2014;32(4):283-288. Available from: https://doi. 1 org/10.1179/2151090314y.0000000008.

4. Aguilar RI, Sánchez FI, Pedraza CG, Guadarrama QLJ. Correlación plantar y maloclusión. Caso clínico. ADM. 12. 2012;69(1):91-94.

5. Solow B TA. Head posture and craniofacial morphology. Am J Phys Antropol. 1976;44(3):417-435.

6. Murrieta J, Arrieta C, Juárez L, Linares C, González M, 13. Meléndez A. Prevalencia de maloclusiones en un grupo de estudiantes universitarios mexicanos y su posible asociación con la edad el sexo y el nivel socioeconómico, 2009. Revista Facultad de Odontologica. 2012;24(1). Available 14 from: https://doi.org/10.18485/beoiber.2018.2.1.11.

7. Urrego, L and Londoño, M and Tamayo, M and Botero, P P, Jimenéz. Perfil epidemiológico de la oclusión dental en escolaresde Envigado, Colombia. Revista Salud Públi- 15. ca. 2011;13(6). Available from: https://doi.org/10.1590/ s0124-00642011000600013.

8. Fajardo J, González L. Prevalencia de Maloclusiones Dentales en escolares de 12 años en Monay-Cuenca 2016. 16. Revista OACTIVA UC Cuenca. 2016;1(2). Available from: https://doi.org/10.31984/oactiva.v1i2.133.

9. Salinas, L and Jiménez-Romero, M, Urgilés C. Maloclusiones dentales en escolares de 12 años en la parro- 17. quia El Sagrario-Cuenca 2016. Salud \& Vida Sipanense. 2017;4(2):58-66. Available from: https://doi.org/10. 26871/killcana_salud.v1i2.72.

10. Astudillo S, González L. Prevalencia de Maloclusiones dentales en escolares de 12 años en la parroquia Bellavista, Cuenca, 2016. Revista Killkana Salud y Bienestar. 2017;1(2):21-26.

1. von Arx UJ. Ortodoncia. Barcelona: Ediciones de la Universitat de Barcelona; 2002. Available from: https: //doi.org/10.21149/spm.v58i5.8182.

2. Talley M Pérez Tejada H KKM. Casuística de maloclusiones Clase I, Clase II y Clase III según Angle en el Departamento de Ortodoncia de la UNAM. Rev Odont Mexicana. 2007;11(4):175-180.

. Pincheira-Garcés C, Thiers-Leal A, Bravo-Sepúlveda E, Olave-Castillo H. Prevalencia de Maloclusiones en Escolares de 6 y 12 Años de Choshuenco - Neltume, Chile. Rev Int J Med Surg Sci. 2014;3(2):829-837.

4. Ramírez-Mendoza J, Rueda-Ventura M, Morales-García M, Gallegos-Ramírez A. Prevalencia de Caries Dental y Maloclusiones en Escolares de Tabasco, México. Rev Horizonte Sanitario. 2012;11(1).

5. Medina C. Prevalencia de maloclusiones dentales en un grupo de pacientes pediátricos. Rev Acta odontol Venez. 2010;48(1). Available from: https://doi.org/10.1002/ajpa. 1330440306.

6. Ruiz-Díaz, A and Guiglione, M, Collante-Benítez C. Prevalencia de maloclusiones en pacientes del servicio de ortodoncia de la Facultad de Odontología UNNE 2013. Rev Facultad de Odontología. 2015;7(1).

7. Manjusha-Jyothindrakumar, K and Nishad, A and Manoj, KM KK. Growth and Development of Dentofacial Complex influenced by Genetic and Environmental Factors using Monozygotic Twins. J Contemp Dent Pract. 2017;18(9):754-758.

Recibido: 3 de noviembre de 2019

Aceptado: 18 de enero de 2020 
